www.jmscr.igmpublication.org

Index Copernicus Value: 79.54

ISSN (e)-2347-176x ISSN (p) 2455-0450

crossrefDOI: https://dx.doi.org/10.18535/jmscr/v7i3.201

\title{
Recurrent Disseminated Abdominal Hydatid Cyst in an Immunosuppressed Individual
}

\author{
Authors \\ Arun $\mathbf{P}^{*}$, Malarvizhi $\mathbf{M}^{2}$, Rajkumar Solomon ${ }^{3}$ \\ ${ }^{1}$ Resident, ${ }^{2}$ Assistant Professor, ${ }^{3}$ Professor \\ Institute of Medical Gastroenterology, Madras Medical College, Chennai, Tamil Nadu \\ *Corresponding Author \\ Arun P \\ Email: arunvani9496@gmail.com
}

\begin{abstract}
Hydatid disease is caused by Echinococcus granulosus and is endemic in cattle and sheep-raising regions of the world. Although it is more common in liver and lung, it also affects brain, spleen and muscle. We are presenting a case of retroviral infection with recurrent intraabdominal hydatid cyst and bone cyst. The rarity of this case is that hydatid cyst is present in liver, spleen, peritoneum, omentum, vertebrae, inguinal region. Patient was treated conservatively with albendazole and praziquantel in view of disseminated disease. The conclusion is recurrence can happen following complete resolution

Keywords: Hydatid cyst, Echinococcus granulosus, Disseminated Abdominal hydatid cyst, Inguinal hydatid cyst, Bone hydatid cyst.
\end{abstract}

\section{Introduction}

Hydatidosis due to Echinococcus (E. granulosus) is a parasitic zoonosis characterized by worldwide distribution especially in Mediterranean countries. It poses a major public health threat in developing countries. The disease commonly affects the liver (> $65 \%$ ) followed by the lungs $(>25 \%)^{[1]}$. Although India is not predominantly a sheep-rearing country, many number of cases have been reported from Tamil Nadu, Andhra Pradesh and other southern states of India ${ }^{[2]}$. Humans are accidental host and do not play a major role in the biological cycle of the parasite. Usual mode of infection is through the ingestion of food contaminated with faeces of dogs and also by direct contact. Common sites affected are the liver $(62 \%)$, the lungs $(26 \%)$, followed by muscles $(6 \%)$, bones (2\%), kidney (1\%), spleen $(2 \%)$, and other sites $(1 \%)^{[3]}$. Liver acts as the primary filter in trapping the embryos which later develop into hydatid cysts in 56-70\% of cases, followed by lungs as the secondary filter in $17-35 \%$ of cases. Some evade from these filters and develop in other organs ${ }^{[4]}$. Disseminated hydatidosis is a rare condition that mainly results from the rupture of a hepatic cyst, with subsequent seeding of protoscolices in the peritoneal cavity ${ }^{5}$. The purpose of this report is to emphasize the fact that hydatidosis can involve any organ of the body and can have varied presentation; therefore, a high degree of suspicion and prolonged treatment is justified in order to prevent fatal complications and chances of recurrence. 


\section{Case Report}

$36 \mathrm{yr}$ old man with history of gradually progressive abdominal distension for 1 month. There was no associated abdominal pain, jaundice, loss of appetite, loss of weight. No history of vomiting, diarrhea was there. Past history of disseminated hyadatid cyst with concomitant retroviral infection was there. Patient was treated with tenofovir, lamivudine, emtricitabine regimen with albendazole 10-15 $\mathrm{mg} / \mathrm{kg}$ for 3 months. Patient did not return for follow up as abdominal distension, pain got completely resolved. $\mathrm{He}$ is a sheep rearer by profession and does not have any addictions. Clinical examination revealed pallor, marfanoid habitus, protruded abdomen. Abdominal examination revealed multiple ill defined nodular firm swellings of varying size in hypochondrium, umbilical region. Massive hepatomegaly with irregular, nodular surface was palpable. Hydatid thrill was elicitable. Genital examination revealed a $2 * 3 \mathrm{~cm}$ round cystic swelling in right inguinal region. Haematological examinations revealed anemia (haemoglobin 10) with mild leucocystosis $\left(10800 / \mathrm{mm}^{3}\right)$.Renal function test and liver function test were normal except for hypoalbuminaemia. Ultrasound examination revealed multiple hyperechoic cystic lesions with daughter cysts in liver and inguinal region. Contrast enhanced Computed Tomography scan showed multiple hypodense cystic lesions of varying size noted in both lobes of liver , largest of being $14 * 8.5 * 13 \mathrm{~cm}$ in the right lobe(CE $2 \mathrm{~A} \& 2 \mathrm{~B}$ ) (figure 1).Some had daughter cyst arranged in periphery giving a racemose appearance. Multiple specks of calcification were noted in spleen. Multiple lesions of varying sizes were noted in mesentry, retroperitoneum (CE 2A,2B,2C) (figure 2), pelvis and small cyst of size $3 * 2 \mathrm{~cm}$ noted in the right inguinal region overlying pubic bone. Numerous lytic lesions were noted in lumbar vertebrae, pelvis and inferior angle of scapula. Xray of pelvis revealed a sclerotic lesion with periosteolytic border (figure 3). ELISA and latex agglutination test came out as negative for hydatidosis. Workup for amoebiasis, tumour markers assay turned out to be negative. Colonoscopy and gastroduodenoscopy workup for malignancy were negative. Old records revealed right lung cyst with disseminated abdominal hydatid cyst. Repeat Computed tomography with basal sections had healed scar with fibrotic strands. Patient was treated with albendazole $(10-15 \mathrm{mg} / \mathrm{kg})$ in two divided doses with praziquantel $600 \mathrm{mg}$ three times daily. Extended course for more than 6 months with review scan after 3 months is planned in view of chance of recurrence.

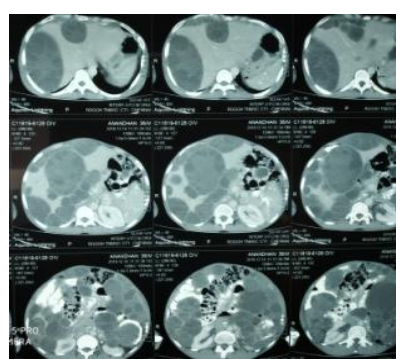

Fig. 1 Hepatic hydatid cyst

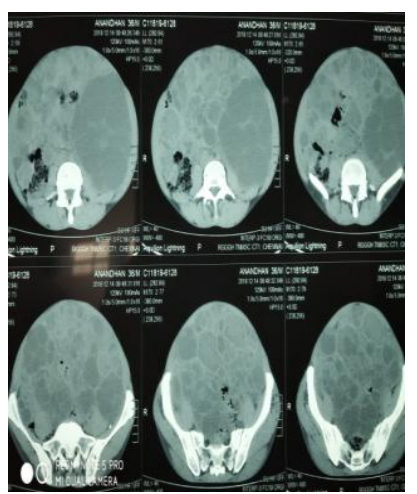

Fig. 2 Mesentric hydatid cyst

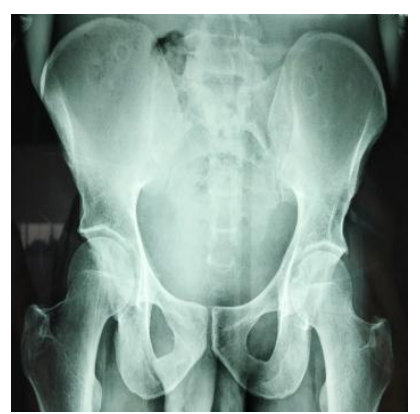

Fig, 3 Hydatid cyst in pelvic bone

\section{Discussion}

Hydatid disease is a parasitic infection caused by Echinococcus granulosus. Hydatid disease (HD) is mostly asymptomatic and diagnosed incidentally as the cyst growth rate is slow and progressive, 
ranging from 1 to $4 \mathrm{~mm}$ in diameter per year. Mostly primary infected individuals will have single cyst, but up to $20 \%-40 \%$ among them can develop multiple cysts $^{[6]}$. Peritoneal hydatidosis is an uncommon manifestation of HD. Secondary peritoneal disease, which accounts for most common form of peritoneal HD, occurs as a result of traumatic rupture of a hepatic, splenic cyst. Spontaneous rupture of intra-abdominal micro cysts within the peritoneum may occur in about $12 \%$ of the cases. Symptoms due to peritoneal hydatid involvement arise commonly from compression due to enlarging abdominal cysts or rupture which may manifest as acute abdominal pain.

Antigenic fluid release into cavity may produce anaphylaxsis. The most useful diagnostic utility would be abdominal ultrasound or CT scan in which lesions appear well defined with or without internal separation $^{[7]}$. The World Health Organization Informal Working Group on Echinococcosis (WHO-IWGE) introduced a standardized classification based on the active-transitionalinactive status of the hydatid cyst as suggested by its ultra sonographic appearance. Cystic echinococcosis 1 (CE1) and CE2 are active cysts having viable protoscolices. CE3 has been subdivided into CE3a (with detached endocyst) and CE3b (mainly solid component with few daughter cysts).CE 4 and CE 5 are inactive degenerated cyst. W. Hosch and his colleagues has shown that calcification within is not restricted to the inactive cyst types CE4 and CE5 but can occur in all stages $\left[{ }^{[8]}\right.$.

Out of the various serological investigations, antibody detection is more sensitive than antigen detection $^{[9]}$. In doubtful cases, ultrasonographyguided fine needle puncture may be an additional diagnostic option. Demonstration of protoscolices or membranes, antigens in the aspirated cyst fluid can confirm the diagnosis. The treatment modality of choice for localized hydatid cysts in liver, lungs, spleen is purely surgical while therapy for disseminated peritoneal hydatidosis is medical. In contrast to monotherapy with either agent, combination therapy with albendazole and praziquantel produced a marked reduction in both the number and viability of cysts ${ }^{[10]}$. Likely role for praziquantel in hydatidosis is in prevention of encystment of protoscolices following any perioperative spillage ${ }^{[11]}$. Surgery was the first therapeutic option for hydatid disease ${ }^{[1]}$. Puncture, aspiration, injection, re-aspiration (PAIR) is a recent and minimally invasive therapeutic technique. It is a percutaneous treatment modality comprising of puncture of cyst with aspiration of cyst fluid followed by injection of hypertonic saline and absolute alcohol, and re-aspiration of the cyst fluid contents (PAIR) under sonographic guidance ${ }^{[12]}$.

Our patient was started on albendazole $400 \mathrm{mg}$ two times daily with praziquantel $600 \mathrm{mg}$ three times daily in view of diessiminated abdominal and bone hydatid cyst. Rarity of the case is accounted by the disseminated hydatid cyst in abdomen, with inguinal cyst and bone cyst. The patient being an immunosuppresed individual accounts for the negative serology and recurrence.

\section{Conclusion}

Disseminated abdominal hydatidosis is rare and uncommon presentation of a common endemic zoonosis. In a immunosuppresed individual as in retroviral infection, chance of dissemination and recurrence is high. Conservative medical management is the treatment protocol for disseminated hydatid disease. Inguinal swelling mimicking hernia along with bone hydatid cyst is very unusual manifestations of hydatidosis.

\section{Acknowledgement}

Sincere thanks to Vimal, Radiology resident,Madras Medical College.

\section{References}

1. Moro P, Schantz PM. Echinococcosis: a review. Int J Infect Dis. 2009; 13: 125-133.

2. Parija SJ. Textbook of medical parasitology. 2nd ed. All India Publishers and Distributors; 2004 [chapter 11], p. 221-29.

3. Topley M, Wilson M. Microbiology and microbial infections: medical parasitology. 
10th ed. Hodder Arnold; 2005 [chapter 33], p. 677-702

4. Ahmad QA, Ahmed MS. Splenic hydatid: a rare presentation of hydatid disease. Annals 2010;16(2):129-31

5. Majbar MA, Souadka A, Sabbah F, Raiss M, Hrora A, Ahallat M. Peritoneal echinococcosis: anatomoclinical features and surgical treatment. World $\mathbf{J}$ Surg. 2012;36:1030-1035

6. Nunnari G, Pinzone MR, Gruttadauria S, Celesia BM, Madeddu G, Malaguarnera G, Pavone P, Cappellani A, Cacopardo B. Hepatic echinococcosis: clinical and therapeutic aspects. World J Gastroenterol. 2012;18:1448-1458

7. A. H. Dachman, P. R. Ros, P. J. Murari, W. W. Olmsted, and J. E. Lichtenstein, "Nonparasitic splenic cysts: a report of 52 cases with radiologic-pathologic correlation," The American Journal of Roentgenology, vol. 147, no. 3, pp. 537-542, 1986.

8. WHO Informal Working Group, "International classification of ultrasound images in cystic echinococcosis for application in clinical and field epidemiological settings," Acta Tropica, vol. 85 , no. 2, pp. 253-261, 2003

9. H. Babba, A. Messedi, S. Masmoudi et al., "Diagnosis of human hydatidosis: comparison between imagery and six serologic techniques," The American Journal of Tropical Medicine and Hygiene, vol. 50, no. 1, pp. 64-68, 1994.

10. D. H. Taylor, D. L. Morris, D. Reffin, and K. S. Richards, "Comparison of albendazole, mebendazole, and praziquantel chemotherapy of Echinococcus multilocularis in a gerbil model," Gut, vol. 30, pp. 1401-1405, 1989.

11. C. H. King and A. A. Mahmoud, "Drugs five years later: praziquantel," Annals of Internal Medicine, vol. 110, no. 4, pp. 290296, 1989.
12. Junghanss $\mathrm{T}$, da Silva AM, Horton J, Chiodini PL, Brunetti E. Clinical management of cystic echinococcosis: state of the art, problems, and perspectives. Am J Trop Med Hyg. 2008;79:301-311. 